in vivo 31 : 905-908 (2017)

doi:10.21873/invivo.11145

\title{
5-Aminolevulinic-acid-mediated Photodynamic Diagnosis Enhances the Detection of Peritoneal Metastases in Biliary Tract Cancer in Mice
}

\author{
TOSHIHIRO KUSHIBIKI ${ }^{1}$, TAKEHIRO NOJI ${ }^{1}$, YUMA EBIHARA ${ }^{1}$, KOJI HONTANI ${ }^{1}$, MASATO ONO ${ }^{1}$, \\ SHOTA KUWABARA ${ }^{1}$, TORU NAKAMURA ${ }^{1}$, TAKAHIRO TSUCHIKAWA ${ }^{1}$, \\ KEISUKE OKAMURA ${ }^{1}$, MASAHIRO ISHIZUKA ${ }^{2}$ and SATOSHI HIRANO ${ }^{1}$ \\ ${ }^{1}$ Department of Gastroenterological Surgery II, Hokkaido University Graduate school of Medicine, Sapporo, Japan; \\ ${ }^{2}$ SBI Pharmaceuticals Co. Ltd., Tokyo, Japan
}

\begin{abstract}
Background/Aim: Previous studies on the accuracy of 5-aminolevulinic-acid-mediated photodynamic diagnosis (5$A L A P D D)$ have been reported for various cancers and brain surgery. However, biliary tract cancer is rare. Therefore, 5-ALA $P D D$ has not been fully evaluated in biliary tract cancers. Small biliary tract cancer lesions such as peritoneal dissemination, liver metastases, and lymph node metastases are negative prognosticators in patients with biliary cancer. The purpose of this exploratory study was to determine if 5-ALA PDD could detect small biliary tract cancer lesions in murine models of biliary cancers. Materials and Methods: Biliary cancer cell lines (TFK-1, HuCCT-1, G415, HuH28, SSP25, RBE, KKU055 and KKU100) and Normal human dermal fibroblast cells were used to evaluate protoporphyrin IX (PpIX) accumulation in vitro. Subcutaneous tumor mice were established using two cell lines (TFK-1 and HuCCT-1). 5-ALA (250 mg/kg) was administered intraperitoneally, and fluorescent 5ALA-PDD was performed 3 $h$ later to evaluate tumoral PpIX accumulation. A murine peritoneal disseminated nodule model was established by intraperitoneal injection of TFK-1 cells. Four weeks later, 5-ALA was administered intraperitoneally, and 5-ALA-PDD was performed $3 \mathrm{~h}$ post administration to evaluate PpIX accumulation in the disseminated nodules. The presence of tumor cells in tumors and nodules was confirmed by haematoxylin and eosin staining. Results: Compared TO non-
\end{abstract}

This article is freely accessible online.

Correspondence to: Prof. Takehiro Noji, Department of Gastroenterological Surgery II, Hokkaido University Graduate School of Medicine, N-15 W-7, Kitaku, Sapporo, 060-8638, Japan. Tel: +81 117067714, Fax:+81 117067158, e-mail: drnoji@med.hokudai.ac.jp

Key Words: 5-Aminolevulinic acid, photodynamic diagnosis, biliary tract cancer. cancerous cell lines, PpIX accumulation was increased in biliary tract cancer cell lines. PpIX accumulation led to a strong fluorescent signal in all subcutaneous tumors. In the murine model of peritoneal dissemination, microdisseminated nodules $(<1 \mathrm{~mm})$ that could not be detected under white light were clearly visible using 5-ALA-PDD. Conclusion: 5-ALA PDD was useful for diagnosis of biliary tract cancer and detection of small peritoneal metastatic lesions in murine models of biliary cancers. Clinical studies and applications of 5-ALA PDD for biliary tract cancer are expected in the future.

Biliary cancers, such as perihilar cholangiocarcinoma, gallbladder carcinoma and intrahepatic cholangiocarcinoma, are one of the most difficult cancers to manage regarding staging and radical resection. Despite these facts, preoperative diagnosis, management, and operative strategies have improved. Major hepatectomy and vascular reconstructions for curative resection of biliary cancers are highly invasive surgeries associated with high morbidity and mortality, with a 5-year survival rate of approximately $40 \%$ (1-6).

Biliary carcinoma exhibits multiple recurrence patterns with distant metastasis such as liver or peritoneal metastases being most common. Therefore, many patients already have small, minimally-invasive, occult liver or peritoneal metastasis before undergoing surgery $(1,7)$. Presently, modalities such as computed tomography and magnetic resonance imaging are inadequate for the detection of small occult lesions. Improved detection of small lesions could inform surgeons to avoid any unnecessary surgeries.

The metabolite of the amino acid 5-aminolevulinic acid (5ALA), termed protoporphyrin IX (PpIX) selectively accumulates in tumors. PpIX emits red fluorescence peaking at $635 \mathrm{~nm}$ under blue-violet light excitation of 400-410 nm (8). In clinical trials, Stummer et al. (9) first demonstrated the use of photodynamic diagnosis (PDD) using 5-ALA to diagnose malignant glioma, intraoperatively. Subsequently, 5-ALA PDD 
use has been evaluated for various cancers and brain surgery $(8,10,11)$. However, biliary tract cancer is rare. Therefore, 5ALA PDD has not been fully evaluated in biliary tract cancers $(12,13)$. 5-ALA PDD could be useful for detecting occult lesions, which are negative prognosticators in patients with biliary cancer, and, therefore, could herald a new era in biliary malignancy management.

The aim of this exploratory study was to evaluate whether 5-ALA PDD would be useful for detecting occult lesions in biliary cancers. First, we examined tumor-specific PpIX accumulation in vitro in biliary tract cancer cell lines. Second, we utilised murine models of biliary tract cancer to determine if small peritoneal disseminated nodules could be detected using 5-ALA PDD.

\section{Materials and Methods}

Cell culture. The biliary tract cancer cell lines KKU055 and KKU100 were purchased from the Japanese Collection of Research Bioresources (JCRB, Osaka, Japan). The biliary tract cancer cell lines HuH28, SSP25 and RBE, were purchased from RIKEN Bio Resource Center (Tokyo, Japan). The biliary tract cancer cell lines TFK-1, HuCCT-1 and G415 were provided by the Cell Resource Center for Biomedical Research, Tohoku University (Sendai, Japan). Normal human dermal fibroblasts (NHDF) were purchased from PromoCell (Heidelberg, Germany). All cells were cultured in appropriate media: RPMI-1640 (Nacalai Tesque, Kyoto, Japan) for TFK-1, HuCCT-1, G415, HuH28, SSP25 and RBE cells and Dulbecco's modified Eagle's medium (Nacalai tesque, Kyoto, Japan) for KKU055, KKU100 and NHDF cells. Media were supplemented with $10 \%$ foetal bovine serum (Cell culture Bioscience, Tokyo, Japan) and $1 \%$ penicillin-streptomycin (Life Technologies, Tokyo, Japan). Cells were maintained at $37^{\circ} \mathrm{C}$ in a $5 \% \mathrm{CO}_{2}$ atmosphere.

PpIX fluorescence measurements. Cells were incubated in medium containing various concentrations of 5ALA for $3 \mathrm{~h}$. Cells were resuspended in a 96-well plate. PpIX accumulation was measured by fluorescence intensity using the IVIS Spectrum imaging system (Caliper Life-Sciences, Hopkinton, MA, USA) at an excitation wavelength of $430 \mathrm{~nm}$ and an emission wavelength of $640 \mathrm{~nm}$. Fluorescence was observed using a Flashlight type Violet LED (SBI Pharmaceuticals Co. Ltd., Tokyo, Japan) and Sharp cut filter (cut-off wavelength, $460 \mathrm{~nm}$; SBI Pharmaceuticals Co. Ltd., Tokyo, Japan).

Murine subcutaneous tumor model. Five-week-old male BALB/c nude mice were purchased from CLEA (Tokyo, Japan). TFK-1 and HuCCT- 1 cells $\left(1 \times 10^{7}\right)$ in $100 \mu \mathrm{L}$ of HBSS (Gibco, Carlsbad, CA). containing Matrigel (Corning, NY, USA) were injected subcutaneously into the back. After 5 weeks, when the average tumor diameter was approximately $8 \mathrm{~mm}, 5$-ALA $(250 \mathrm{mg} / \mathrm{kg})$ was administrated intraperitoneally.

Establishment of the murine model of peritoneal metastasis. TFK-1 cells $\left(5 \times 10^{5}\right)$ were injected into the peritoneal cavity under general anaesthesia. After 4 weeks, 5-ALA hydrochloride (SBI Pharmaceuticals) was administered intraperitoneally at a dose of 250 $\mathrm{mg} / \mathrm{kg}$ body weight. Three $\mathrm{h}$ post administration, mice were euthanized, and laparotomies were performed. Peritoneal metastatic nodules were observed under white light and fluorescence imaging (described above).
This study was approved by the Institutional Animal Care and Use Committee at the Hokkaido University and was performed according to institutional guidelines.

\section{Results}

Accumulation of PpIX in biliary cancer cell lines. We investigated whether PpIX accumulation in TFK-1, HuCCT1 , and G415 cell lines and NHDF cells (control) could be detected using 5-ALA. A range of 5-ALA concentrations (0.5, 1 , and $10 \mathrm{mM}$ ) were evaluated in various cell numbers (1 to $\left.1 \times 10^{5}\right)$. At 5-ALA concentrations above $1 \mathrm{mM}$, the fluorescence intensities of biliary tract cancer cell lines were greater compared with that in the NHDF cells (Figure 1). Similar results were obtained for the RBE, HuH28, HSP25, KKU25, and KKU100 cells (data not shown).

Murine subcutaneous tumor model. Five subcutaneous tumor model mice were prepared using two biliary tract cancer cell lines (TFK-1 and HuCCT-1) to determine if PpIX fluorescence could be detected after 5-ALA administration. PpIX accumulation led to a strong fluorescent signal in all mice (Figure 2). Subcutaneous tumors were histopathologically confirmed to be cancerous (data not shown). Fluorescence was detected in both solid and cystic tumors (data not shown).

Murine peritoneal dissemination model. 5-ALA PDD was conducted using a TFK-1 murine model of liver metastasis and peritoneal dissemination. Ten mice were euthanised $3 \mathrm{~h}$ post 5-ALA ( $250 \mathrm{mg} / \mathrm{kg}$ ) administration. Figure 3A shows a strong fluorescent signal for liver metastases (approximately $1.5 \mathrm{~mm}$ diameter) indicating marked PpIX accumulation. PpIX accumulation was also detected in small peritoneal lesions $(<1 \mathrm{~mm})$ (Figure 3B). Liver metastases and peritoneal lesions were histopathologically confirmed as adenocarcinomas (Figure $3 \mathrm{~A}$ and $\mathrm{B}$, respectively). PpIX accumulates in the small and large intestines; therefore, peritoneal disseminations on the surface of these organs could not be detected using 5-ALA PDD (data not shown).

\section{Discussion}

In the present study, PpIX accumulation was significantly greater in biliary tract cancer cells compared with that in control NHDF cells. Furthermore, fluorescent visualisation of PpIX accumulation was possible in murine subcutaneous tumor models of biliary cancer in both solid and cystic tumors. These findings suggest that 5-ALA PDD could be applied to various tumor types. Most importantly, we demonstrated that 5-ALA PDD could be used successfully to detect small peritoneal disseminated lesions originating from biliary malignancies.

The present exploratory study forms the basis for a potential clinical application of 5-ALA PDD in detecting biliary tract cancer disseminations in patients. However, several issues must 

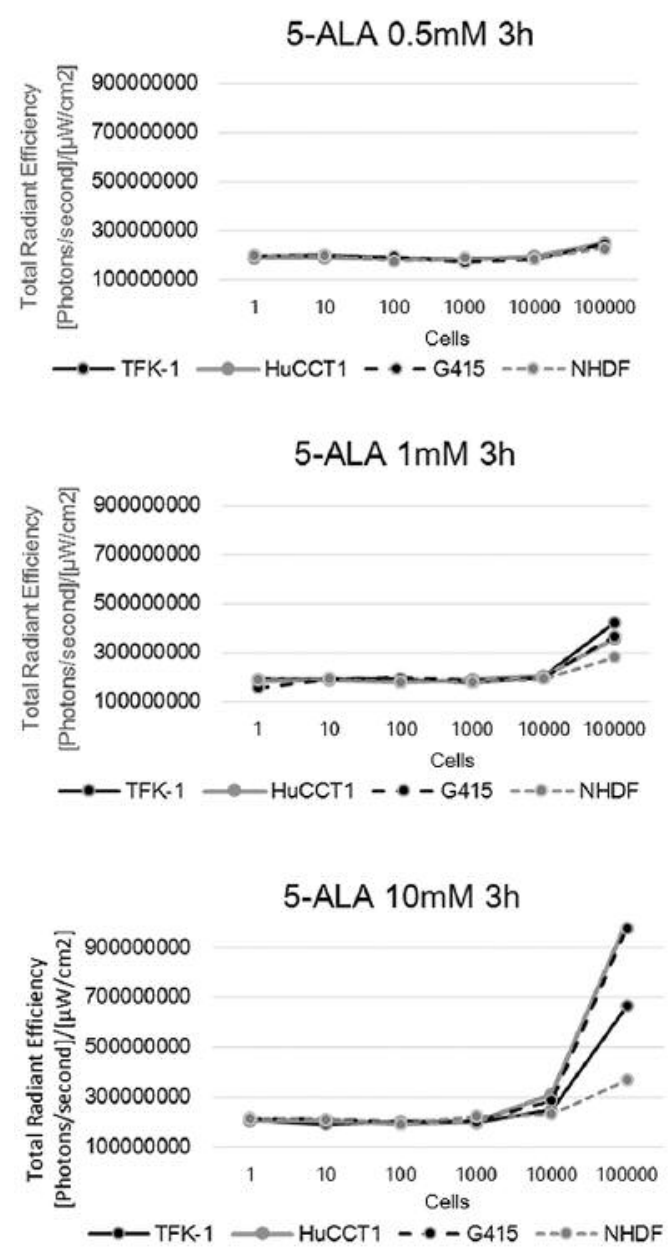

Figure 1. Fluorescent intensities of 5-ALA PDD in vitro. Fluorescence was quantitated using the IVIS Spectrum imaging system.

be resolved before the technique can be evaluated clinically. Foremost would be the issue of light penetration depth. Blue light excitation is used for 5-ALA PDD. However, blue light penetration depth is shallower than that of red light (mean, 0.6 $\mathrm{cm})(14,15)$, and blue light penetration depth is significantly affected by adipose tissue. Therefore, it would be difficult to observe tumors originating outside the bile duct, but 5-ALA PDD might be useful for detection of exposed tumor cells such as those on the resected stump of the bile duct.

A further issue could be the differential diagnosis of inflammation. Previous studies have shown that PpIX accumulates at inflammation sites $(16,17)$. More than half of the patients undergoing surgery for bile duct surface tumors also undergo preoperative biliary drainage, which can lead to significant inflammation, posing a problem for 5-ALA PDD. Furthermore, previous studies have also demonstrated that 5-ALA is excreted in the bile, which while useful for a diagnosis of intraoperative bile leakage, might lead to false positives for bile duct cut surface $(18,19)$.

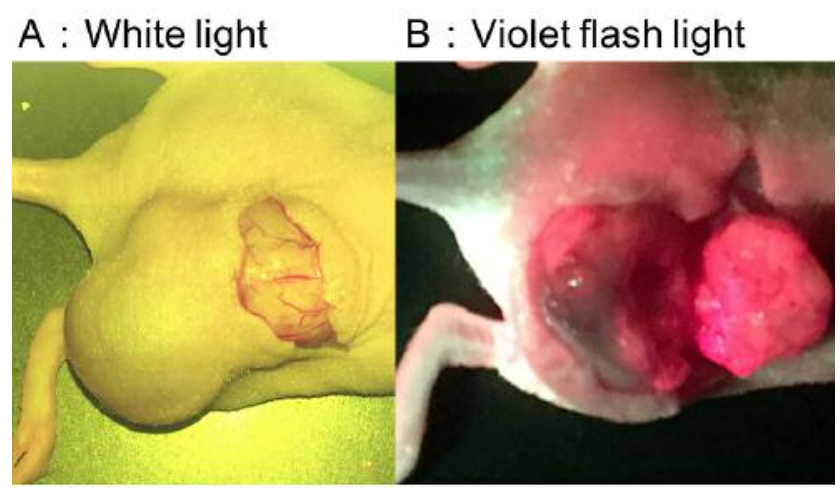

Figure 2. Fluorescent imaging of TFK-1 subcutaneous tumors in mice. Strong fluorescence was observed in the bisected tumor.

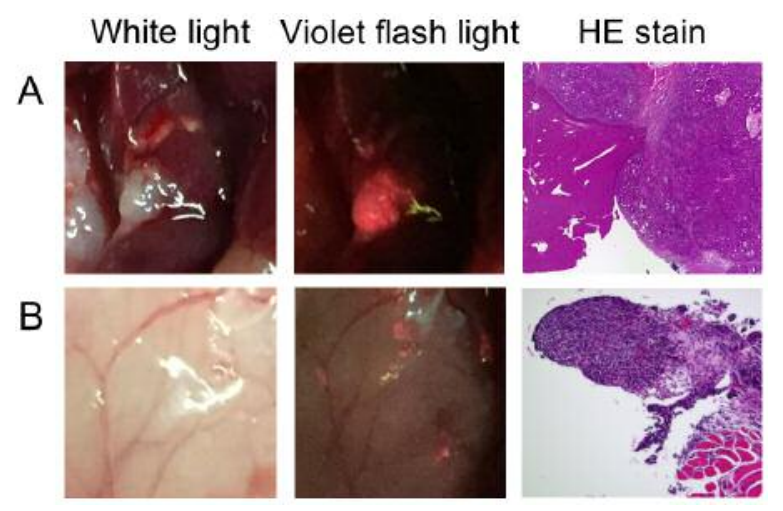

Figure 3. Disseminated nodule imaging. A: Liver metastatic tumor nodule (TFK-1 cells) in mice. The nodule diameter was approximately $1.5 \mathrm{~mm}$. B: Microdisseminated nodules (TFK-1 cells) in mice. The nodule diameter was $<1 \mathrm{~mm}$. Right-side panels show corresponding haematoxylin and eosin staining to confirm the tumors as adenocarcinomas.

Previous clinical studies have shown that 5-ALA PDD could detect peritoneal disseminated lesions from both gastric and ovarian cancers (20-22). Similarly, in the present study, it was possible to detect small disseminated lesions originating from biliary tract cancer. However, as seen in the present and previous studies that utilized murine models, non-specific PpIX accumulation occurs in other organs, particularly the small and large intestines (23). Therefore, we could not determine if microdisseminated lesions were present on the surface of these organs.

\section{Conclusion}

5-ALA PDD was useful for detecting biliary tumors, liver metastases, and small peritoneal disseminated lesions in murine models of biliary tract cancers. Studies to evaluate the clinical application of 5-ALA PDD in patients with biliary tract cancer are expected in the future. 


\section{Conflicts of Interest}

The Authors have no conflicts of interest to declare. The 5-ALA used in this study was gifted by SBI Pharmaceuticals Co. Ltd.

\section{References}

1 Ebata T, Ercolani G, Alvaro D, Ribero D, Di Tommaso L and Valle JW: Current status on cholangiocarcinoma and gallbladder cancer. Liver Cancer 6(1): 59-65, 2016.

2 Igami T, Ebata T, Yokoyama $\mathrm{Y}$, Sugawara G, Mizuno T, Yamaguchi J, Shimoyama $\mathrm{Y}$ and Nagino M: Combined extrahepatic bile duct resection for locally advanced gallbladder carcinoma: Does it work? World J Surg 39(7): 1810-1817, 2015.

3 Miyakawa S, Ishihara S, Horiguchi A, Takada T, Miyazaki M and Nagakawa T: Biliary tract cancer treatment: 5,584 results from the biliary tract cancer statistics registry from 1998 to 2004 in japan. J Hepatobiliary Pancreat Surg 16(1): 1-7, 2009.

4 Noji T, Tsuchikawa T, Okamura K, Tanaka K, Nakanishi Y, Asano T, Nakamura T, Shichinohe T and Hirano S: Concomitant hepatic artery resection for advanced perihilar cholangiocarcinoma: A case-control study with propensity score matching. J Hepatobiliary Pancreat Sci 23(7): 442-448, 2016.

5 Saito H, Noji T, Okamura K, Tsuchikawa T, Shichinohe T and Hirano S: A new prognostic scoring system using factors available preoperatively to predict survival after operative resection of perihilar cholangiocarcinoma. Surgery 159(3): 842-851, 2016.

6 Wiggers JK, Groot Koerkamp B, Cieslak KP, Doussot A, van Klaveren D, Allen PJ, Besselink MG, Busch OR, D'Angelica MI, DeMatteo RP, Gouma DJ, Kingham TP, van Gulik TM and Jarnagin WR: Postoperative mortality after liver resection for perihilar cholangiocarcinoma: Development of a risk score and importance of biliary drainage of the future liver remnant. J Am Coll Surg 223(2): 321-331e321, 2016.

7 Nakanishi Y, Kondo S, Zen Y, Yonemori A, Kubota K, Kawakami H, Tanaka E, Hirano S, Itoh T and Nakanuma Y: Impact of residual in situ carcinoma on postoperative survival in 125 patients with extrahepatic bile duct carcinoma. J Hepatobiliary Pancreat Sci 17(2): 166-173, 2010.

8 Takahashi K, Ikeda N, Nonoguchi N, Kajimoto Y, Miyatake S, Hagiya $Y$, Ogura S, Nakagawa $H$, Ishikawa $T$ and Kuroiwa T: Enhanced expression of coproporphyrinogen oxidase in malignant brain tumors: Cpox expression and 5-ala-induced fluorescence. Neuro Oncol 13(11): 1234-1243, 2011.

9 Stummer W, Stocker S, Wagner S, Stepp H, Fritsch C, Goetz C, Goetz AE, Kiefmann R and Reulen HJ: Intraoperative detection of malignant gliomas by 5-aminolevulinic acid-induced porphyrin fluorescence. Neurosurgery 42(3): 518-525, 1998.

10 Fritsch C, Lang K, Neuse W, Ruzicka T and Lehmann P: Photodynamic diagnosis and therapy in dermatology. Skin Pharmacol Appl Skin Physiol 11(6): 358-373, 1998.

11 Denzinger S, Burger M, Walter B, Knuechel R, Roessler W, Wieland WF and Filbeck T: Clinically relevant reduction in risk of recurrence of superficial bladder cancer using 5-aminolevulinic acid-induced fluorescence diagnosis: 8-year results of prospective randomized study. Urology 69(4): 675-679, 2007.

12 Zoepf T, Jakobs R, Rosenbaum A, Apel D, Arnold JC and Riemann JF: Photodynamic therapy with 5-aminolevulinic acid is not effective in bile duct cancer. Gastrointest Endosc 54(6): 763-766, 2001.
13 Chung CW, Kim CH, Lee HM, Kim DH, Kwak TW, Chung KD, Jeong YI and Kang DH: Aminolevulinic acid derivatives-based photodynamic therapy in human intra- and extrahepatic cholangiocarcinoma cells. Eur J Pharm Biopharm 85(3 Pt A): 503-510, 2013.

14 Webber J, Herman M, Kessel D and Fromm D: Current concepts in gastrointestinal photodynamic therapy. Ann Surg 230(1): 1223, 1999.

15 Braathen LR, Szeimies RM, Basset-Seguin N, Bissonnette R, Foley P, Pariser D, Roelandts R, Wennberg AM, Morton CA and International Society for Photodynamic Therapy in D: Guidelines on the use of photodynamic therapy for nonmelanoma skin cancer: An international consensus. International society for photodynamic therapy in dermatology, 2005. J Am Acad Dermatol 56(1): 125$143,2007$.

16 Messmann H, Knuchel R, Baumler W, Holstege A and Scholmerich J: Endoscopic fluorescence detection of dysplasia in patients with barrett's esophagus, ulcerative colitis, or adenomatous polyps after 5-aminolevulinic acid-induced protoporphyrin ix sensitization. Gastrointest Endosc 49(1): 97-101, 1999.

17 Koizumi N, Harada Y, Murayama Y, Harada K, Beika M, Yamaoka Y, Dai P, Komatsu S, Kubota T, Ichikawa D, Okamoto $\mathrm{K}$, Yanagisawa A, Otsuji E and Takamatsu T: Detection of metastatic lymph nodes using 5-aminolevulinic acid in patients with gastric cancer. Ann Surg Oncol 20(11): 3541-3548, 2013.

18 Inoue Y, Imai Y, Fujii K, Hirokawa F, Hayashi M and Uchiyama $\mathrm{K}$ : The utility of 5-aminolevulinic acid-mediated photodynamic diagnosis in the detection of intraoperative bile leakage. Am J Surg 213(6): 1077-1082, 2016.

19 Inoue Y, Tanaka R, Komeda K, Hirokawa F, Hayashi M and Uchiyama K: Fluorescence detection of malignant liver tumors using 5-aminolevulinic acid-mediated photodynamic diagnosis: Principles, technique, and clinical experience. World J Surg 38(7): 1786-1794, 2014

20 Kishi K, Fujiwara Y, Yano M, Inoue M, Miyashiro I, Motoori M, Shingai T, Gotoh K, Takahashi H, Noura S, Yamada T, Ohue $\mathrm{M}$, Ohigashi $\mathrm{H}$ and Ishikawa $\mathrm{O}$ : Staging laparoscopy using alamediated photodynamic diagnosis improves the detection of peritoneal metastases in advanced gastric cancer. J Surg Oncol 106(3): 294-298, 2012.

21 Kishi K, Fujiwara Y, Yano M, Motoori M, Sugimura K, Ohue M, Noura S, Marubashi S, Takahashi H and Sakon M: Diagnostic laparoscopy with 5-aminolevulinic-acid-mediated photodynamic diagnosis enhances the detection of peritoneal micrometastases in advanced gastric cancer. Oncology 87(5): 257-265, 2014.

22 Hillemanns P, Wimberger P, Reif J, Stepp H and Klapdor R: Photodynamic diagnosis with 5-aminolevulinic acid for intraoperative detection of peritoneal metastases of ovarian cancer: A feasibility and dose finding study. Lasers Surg Med 49(2): 169-176, 2017.

23 Johansson J, Berg R, Svanberg K and Svanberg S: Laser-induced fluorescence studies of normal and malignant tumour tissue of rat following intravenous injection of delta-amino levulinic acid. Lasers Surg Med 20(3): 272-279, 1997. 